\title{
Density functional triple jumping
}

\author{
Jia Deng, Andrew T. B. Gilbert and Peter M. W. Gill* \\ Received 16th April 2010, Accepted 19th June 2010 \\ DOI: $10.1039 /$ c0cp00242a
}

We propose a density functional perturbative scheme to approximate the energy of a high-level DFT calculation at a significantly reduced cost. Our approach involves performing a primary SCF calculation using a crude functional, basis set and quadrature grid, followed by a single step using a more sophisticated secondary functional, basis and grid. Unlike the earlier dual-level DFT approach of Nakajima and Hirao, we use Roothaan diagonalization instead of perturbation theory to incorporate the effects of the secondary basis set. We show that energies at the popular $\mathrm{B} 3 \mathrm{LYP} / 6-311+\mathrm{G}(3 d f, 2 p) /(75,302)$ level can be accurately estimated from primary calculations at the relatively economical BLYP/6-31G(d)/SG-0 level.

\section{Introduction}

Density functional theory (DFT) $)^{1,2}$ has a number of advantages over traditional wavefunction-based methods: it is conceptually simpler, more easily implemented, and usually less computationally demanding. Moreover, DFT often accounts well for energetic, structural and vibrational properties of molecules and, for this reason, it has become a useful tool for experimental chemists of all types. Its accuracy, however, depends critically on the exchange-correlation (XC) functional, basis set and quadrature grid and, as a general rule, greater accuracy incurs a greater computational cost. As interest in large biochemical systems grows, it is important to develop methods that boast both high accuracy and low cost.

DFT calculations using pure (local or gradient-corrected) functionals with small basis sets and small grids are computationally inexpensive. Such calculations require only the Coulomb contributions to the Kohn-Sham matrix and these can be evaluated in $O\left(n^{2}\right)$ cost (where $n$ is the basis set size) using efficient two-electron integral methods ${ }^{3,4}$ and, even more cheaply, by using modern algorithms such as the Continuous Fast Multipole Method (CFMM), ${ }^{5} \mathrm{KWIK}^{6}$ and the Fourier Transform Coulomb (FTC) method., ${ }^{7,8}$ The most timeconsuming step in an efficiently implemented pure DFT calculation is then the evaluation of the various $\mathrm{XC}$ contributions, ${ }^{8}$ the cost of which scales with the size of the quadrature grid.

Although inexpensive, pure DFT calculations lack the accuracy of those that use hybrid functionals (i.e. those that include a fraction of exact Fock exchange). If combined with a large basis set and quadrature grid, hybrid DFT can often produce results that are in very good agreement with experiment but, unfortunately, the inclusion of exact exchange introduces non-locality into the $\mathrm{XC}$ potential and precludes the use of fast techniques such as the CFMM. This means that calculations using hybrid functionals are significantly slower than those using pure functionals, a weakness that has restricted their application to relatively small systems.

Research School of Chemistry, Australian National University, Canberra ACT 0200, Australia.E-mail: peter.gill@anu.edu.au; Tel: 61261254258
In this Paper, we propose Density Functional Perturbation Corrections (DFPC) which can be exploited to combine the low cost of pure DFT calculations using small bases and grids with the high accuracy of hybrid DFT calculations using large bases and grids. Our method is motivated by the dual-level DFT method of Nakajima and Hirao $^{9}$ and the dual grid scheme of Tozer et al. ${ }^{10}$ We combine these with dual basis ideas $^{11-19}$ to obtain a triple perturbation in the functional, basis and grid dimensions.

\section{Theory}

DFPC embraces a family of perturbative corrections that can be applied after a self-consistent KS calculation. It provides a general framework for dual-level DFT calculations that allows jumping in the functional, basis and grid dimensions. Before considering the general case we will outline a dual-basis DFT calculation using the HFPC $^{18,19}$ scheme and, in doing so, introduce our notation.

In practice, DFT calculations are performed in a finite basis of $n$ functions $\left\{\chi_{\mu}\right\}$ using a variational formalism. ${ }^{20}$ When converged, this yields a density matrix, $\mathbf{P}$, and corresponding density

$$
\rho=\sum_{\mu \nu} P_{\mu \nu} \chi_{\mu} \chi_{\nu}
$$

This density gives rise to the coulomb potential

$$
V_{\mathrm{J}}=\int \frac{\rho\left(\mathbf{r}^{\prime}\right)}{\left|\mathbf{r}-\mathbf{r}^{\prime}\right|} \mathrm{d} \mathbf{r}^{\prime}
$$

and, along with the $\mathrm{XC}$ energy functional

$$
E_{\mathrm{xc}}[\rho]=\int \rho(\mathbf{r}) \varepsilon_{\mathrm{xc}}[\rho] \mathrm{d} \mathbf{r}
$$

the $\mathrm{XC}$ potential

$$
v_{\mathrm{xc}}=\varepsilon_{\mathrm{xc}}[\rho]+\rho \frac{\delta \varepsilon_{\mathrm{xc}}[\rho]}{\delta \rho}
$$

where $\varepsilon_{\mathrm{xc}}$ is the XC energy density. These potentials can be used to build a KS matrix in a larger secondary basis of $N$ functions $\left\{\chi_{a}\right\}$

$$
F_{a b}=H_{a b}^{\mathrm{core}}+J_{a b}+F_{a b}^{\mathrm{xc}}\left[v_{\mathrm{xc}}\right]
$$


where $H_{a b}^{\text {core }}$ is the core Hamiltonian matrix in the secondary basis, and

$$
\begin{aligned}
J_{a b} & =\left\langle\chi_{a}\left|V_{J}\right| \chi_{b}\right\rangle \\
F_{a b}^{\mathrm{xc}}\left[v_{\mathrm{xc}}\right] & =\left\langle\chi_{a}\left|v_{\mathrm{xc}}\right| \chi_{b}\right\rangle
\end{aligned}
$$

The integrals that arise in (7) cannot usually be evaluated in closed form and, therefore, quadrature must be used. We can write the quadrature-dependent $\mathrm{XC}$ matrix as

$$
F_{a b}^{\mathrm{xc}}\left[v_{\mathrm{xc}}, G\right]=\sum_{i \in G} w_{i} \chi_{a}\left(\mathbf{r}_{i}\right) v_{\mathrm{xc}}\left(\mathbf{r}_{i}\right) \chi_{b}\left(\mathbf{r}_{i}\right)
$$

where $\mathbf{r}_{i}$ and $w_{i}$ are the roots and weights associated with the quadrature grid $G$. It follows that the KS matrix is also dependent on both the functional and grid, and we can make this dependence explicit by writing $F_{a b}\left[v_{\mathrm{xc}}, G\right]$. This new KS matrix can be diagonalised once to yield an updated (but non-self-consistent) density matrix, $\tilde{\mathbf{P}}$, and corresponding density

$$
\tilde{\rho}=\sum_{a b} \tilde{P}_{a b} \chi_{a} \chi_{b}
$$

Clearly, $\tilde{\rho}$ depends on both the functional and grid used in the KS matrix formation and, where necessary, this dependence can be made explicit by writing $\tilde{\rho}\left[v_{\mathrm{xc}}, G\right]$. For functionals within the generalised gradient approximation (GGA), the total electronic energy can be evaluated via

$$
\begin{aligned}
E\left[\tilde{\rho}, v_{\mathrm{xc}}, G\right]= & \sum_{a b}^{N} \tilde{P}_{a b} H_{a b}^{\mathrm{core}}+\frac{1}{2} \sum_{a b c d}^{N} \tilde{P}_{a b} \tilde{P}_{c d}(a b \mid c d) \\
& +\sum_{i \in G} w_{i} \tilde{\rho}\left(\mathbf{r}_{i}\right) \varepsilon_{\mathrm{xc}}\left(\mathbf{r}_{i}\right)
\end{aligned}
$$

Similar expressions exist for hybrid and meta-GGA functionals. This expression is analogous to the dual-basis energy using the HFPC $[1]^{18}$ scheme and we note that, unlike other dual-basis schemes, it includes contributions that are quadratic in the updated density matrix $\tilde{\mathbf{P}}$.

We are now in a position to be able to define the DFPC energy in its most general form, that is, we allow for changes in the functional, basis and grid. Conceptually, this can be viewed as a "triple jump" across the diagonal of a cube, as shown in Fig. 1. Within this framework several other cases which traverse different edges of this cube can also be defined.

We denote the primary functional and grid used in the SCF calculation by $v_{\mathrm{xc}}^{\mathrm{P}}$ and $G^{\mathrm{P}}$ and the secondary functional and grid used in the final energy evaluation by $v_{\mathrm{xc}}^{\mathrm{S}}$ and $G^{\mathrm{S}}$. If we vary only the basis, then $v_{\mathrm{xc}}^{\mathrm{P}}=v_{\mathrm{xc}}^{\mathrm{S}}=v_{\mathrm{xc}}$ and $G^{\mathrm{P}}=G^{\mathrm{S}}=G$ and we obtain our dual-basis energy

$$
E^{\mathrm{DB}}=E\left[\tilde{\rho}\left[v_{\mathrm{xc}}, G\right], v_{\mathrm{xc}}, G\right]
$$

If we vary only the functional, then $\left\{\chi_{a}\right\}=\left\{\chi_{\mu}\right\}$ and $G^{\mathrm{P}}=G^{\mathrm{S}}=G$ and we obtain the dual-functional energy

$$
\begin{aligned}
& E^{\mathrm{DF}(\mathrm{A})}=E\left[\rho, v_{\mathrm{xc}}^{\mathrm{S}}, G\right] \\
& E^{\mathrm{DF}(\mathrm{B})}=E\left[\tilde{\rho}\left[v_{\mathrm{xc}}^{\mathrm{S}}, G\right], v_{\mathrm{xc}}^{\mathrm{S}}, G\right]
\end{aligned}
$$

The dual-functional energy comes in two flavours, DF(A) and $\mathrm{DF}(\mathrm{B})$, which differ in whether or not the KS matrix is rebuilt using the secondary functional.

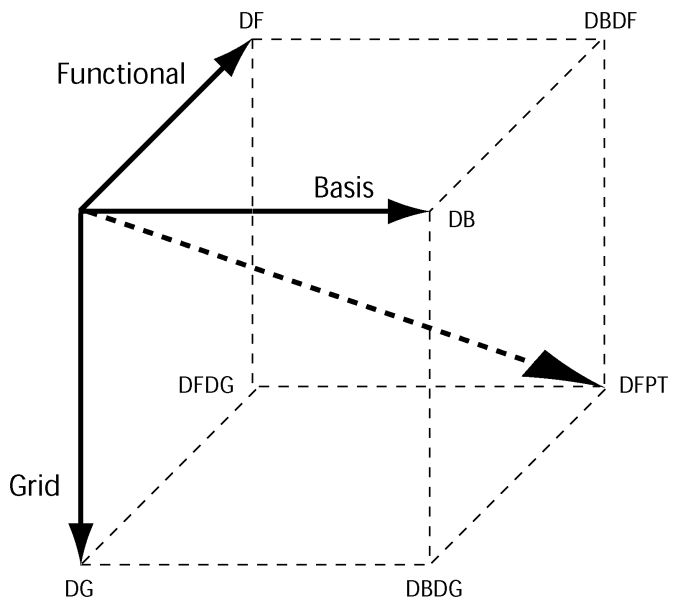

Fig. 1 Density functional perturbation corrections (DFPC) can be viewed as moving along the edges of a cube whose directions represent the functional, basis and grid.

If we vary only the grid, then $\left\{\chi_{a}\right\}=\left\{\chi_{\mu}\right\}$ and $v_{\mathrm{xc}}^{\mathrm{P}}=v_{\mathrm{xc}}^{\mathrm{S}}=v_{\mathrm{xc}}$ and we obtain the dual-grid energy

$$
\begin{aligned}
E^{\mathrm{DG}(\mathrm{A})} & =E\left[\rho, v_{\mathrm{xc}}, G^{\mathrm{S}}\right] \\
E^{\mathrm{DG}(\mathrm{B})} & =E\left[\tilde{\rho}\left[v_{\mathrm{xc}}, G^{\mathrm{S}}\right], v_{\mathrm{xc}}, G^{\mathrm{S}}\right]
\end{aligned}
$$

As in the dual-functional case, the dual-grid energy also comes in two flavours depending on whether or not the KS matrix is rebuilt before the energy evaluation.

We now consider varying two of our three quantities. These levels of theory traverse two edges of the cube in Fig. 1 and we will refer to these as "double jump" methods.

$$
\begin{aligned}
& E^{\mathrm{DFDB}}=E\left[\tilde{\rho}\left[v_{\mathrm{xc}}^{\mathrm{S}}, G\right], v_{\mathrm{xc}}^{\mathrm{S}}, G\right] \\
& E^{\mathrm{DBDG}}=E\left[\tilde{\rho}\left[v_{\mathrm{xc}}^{\mathrm{S}}, G^{\mathrm{P}}\right], v_{\mathrm{xc}}^{\mathrm{S}}, G^{\mathrm{S}}\right] \\
& E^{\mathrm{DFDG}}=E\left[\tilde{\rho}\left[v_{\mathrm{xc}}^{\mathrm{S}}, G^{\mathrm{P}}\right], v_{\mathrm{xc}}^{\mathrm{S}}, G^{\mathrm{S}}\right]
\end{aligned}
$$

For reasons that will become clear later, when we jump in multiple dimensions we always use $v_{\mathrm{xc}}^{\mathrm{S}}$ and $G^{\mathrm{P}}$ when building the KS matrix.

Finally, we can consider changing all three quantities to obtain the DFPC energy

$$
E^{\mathrm{DFPC}}=E\left[\tilde{\rho}\left[v_{\mathrm{xc}}^{\mathrm{S}}, G^{\mathrm{P}}\right], v_{\mathrm{xc}}^{\mathrm{S}}, G^{\mathrm{S}}\right]
$$

It is worth emphasizing that $\tilde{\rho}$ is expressed in the primary basis in (18), but in the secondary basis in (19).

A DFPC calculation is completely defined by six variables and we propose the notation

$$
\left\{X^{\mathrm{P}}, X^{\mathrm{S}}\right\} /\left\{B^{\mathrm{P}}, B^{\mathrm{S}}\right\} /\left\{G^{\mathrm{P}}, G^{\mathrm{S}}\right\}
$$

where $X, B$ and $G$ refer to the $\mathrm{XC}$ functional, the basis set and the grid, respectively, and the superscripts $\mathrm{P}$ and $\mathrm{S}$ distinguish between the primary and secondary levels.

\section{Results and discussion}

To test the efficacy of our approach, we chose the popular $\mathrm{B} 3 \mathrm{LYP} / 6-311+\mathrm{G}(3 d f, 2 p) /(75,302)$ model as our secondary level and all of the errors discussed below are measured relative to this benchmark. Here, and elsewhere in the paper, 
we use $\left(N_{r}, N_{\Omega}\right)$ to denote the molecular quadrature scheme ${ }^{21}$ that pairs the $N_{r}$-point Euler-Maclaurin radial grid ${ }^{22}$ with the $N_{\Omega}$-point Lebedev angular grid $^{23}$ and we note that a pruned $(75,302)$ is the "Fine Grid" in the Gaussian 09 package. ${ }^{24}$

All molecular structures were optimized at the B3LYP/ 6-31G $(2 d f, p) / \mathrm{SG}-1$ level. Incremental $\mathrm{DFT}^{25}$ was switched off, and all SCF calculations were converged to a DIIS error of $10^{-7}$. All calculations were performed using a development version of the Q-Chem 4.0 quantum chemistry package. ${ }^{26}$

Several primary levels (functional, basis set and grid) were explored, and each was applied to the 257 neutral molecules in the G3 dataset. ${ }^{27}$ To assess each primary level, we found its mean absolute error, maximum absolute error, worst case molecule, and number of outliers (NO), relative to the benchmark energies. (An outlier is a molecule whose absolute error exceeds $4 \mathrm{~kJ} / \mathrm{mol}$.) These statistics are collected in Table 1 .

\subsection{Functional jumping}

We begin our DFPC exploration by studying cases in which the primary and secondary levels differ only in the functional.

Three primary functionals were investigated: SVWN, ${ }^{28,29}$ BLYP $^{30,31}$ and EDF1. ${ }^{32}$ Their mean errors relative to B3LYP range from $5424 \mathrm{~kJ} / \mathrm{mol}$ for $\mathrm{SVWN}$ to $112 \mathrm{~kJ} / \mathrm{mol}$ for EDF1. Although these are large, there is usually significant cancellation when considering chemical reactions. Our purpose in showing them here is to demonstrate that the dual functional schemes are able to remove almost all of these errors.

The simple DF(A) scheme produces huge improvements, the BLYP mean error, for example, drops from 168 to $5.9 \mathrm{~kJ} / \mathrm{mol}$ but, unfortunately, the number of outliers (178 for BLYP) is still unacceptably large for chemical purposes. The DF(B) scheme, however, is several times more accurate, reducing the BLYP mean error to just $0.4 \mathrm{~kJ} / \mathrm{mol}$ and removing all the outliers. The $\mathrm{DF}(\mathrm{B})$ errors for the SVWN functional are still large and we therefore do not recommend using a local density functional if the target secondary functional is a hybrid.

Dual functional schemes with gradient-corrected primary functionals seem promising. The two functionals considered here are comparable; EDF1 slightly outperforms BLYP for the $\mathrm{DF}(\mathrm{A})$ scheme and vice versa for $\mathrm{DF}(\mathrm{B})$. These results suggest that dual functional approaches are insensitive to the choice of gradient-corrected functional in the primary calculation.

\subsection{Basis jumping}

Next, we turn to cases in which the primary and secondary levels differ only in the basis set.

Three primary basis sets were investigated: 6-31G, 6-31G $(d)$ and $6-311 \mathrm{G}(d)$. Their mean errors relative to $6-311+\mathrm{G}(3 d f, 2 p)$ range from 402 to $78 \mathrm{~kJ} / \mathrm{mol}$ and almost all of their errors exceed $4 \mathrm{~kJ} / \mathrm{mol}$. Our dual-basis perturbative approach significantly reduces these errors, the mean falling to $26 \mathrm{~kJ} / \mathrm{mol}$ for 6-31G and below $2 \mathrm{~kJ} / \mathrm{mol}$ for the polarized basis sets. It is interesting to contrast the performances of the $6-31 \mathrm{G}(d)$ and 6-311G $(d)$ bases. Although the former has fewer functions and a much larger primary mean error, it yields superior DFPC energies. This unexpected result is also seen in the HFPC method $^{19}$ and recalls the claim by Schaefer and $\mathrm{Grev}^{33}$ that the quality of the 6-311G basis is questionable.

There is a significant improvement in the DB energies when polarization functions are included in the primary basis. These functions are required to model the orbitals adequately in hypervalent molecules such as $\mathrm{PCl}_{5}, \mathrm{PF}_{5}$ and $\mathrm{SF}_{6}$, which consistently feature among the outliers. Unpolarized basis sets yield poor primary potentials for such systems and our perturbative DB approach is unable to recover satisfactory orbitals from this poor starting point. We therefore recommend the use of primary basis sets with at least one set of $d$ functions for such systems. The $6-31 \mathrm{G}(d) \rightarrow 6-311+\mathrm{G}(3 d f, 2 p)$ pairing is particularly attractive due to the large ratio between the sizes of the two basis sets.

\subsection{Grid jumping}

Although an impressive selection of small grids can be constructed by pairing small radial and angular grids, a lack of systematic pruning ${ }^{21}$ means that none of these is optimal. To test our dual grid approach, we therefore chose SG-0, ${ }^{34,35}$ a small, pruned, standard grid as our primary grid. It uses roughly 1400 grid points per atom and yields grid errors that are roughly twice as large as the popular SG-1 grid. ${ }^{21}$

It is clear from the results in Table 1 that even this very small primary grid introduces negligible errors into the perturbative calculations. The primary calculations using this grid have

Table 1 Mean absolute errors (MEAN, kJ/mol), maximum absolute errors (MAX, kJ/mol), worst case (WC) and number of outliers (NO) for DFT and DFPC calculations on the neutral G3 molecules. $2^{\circ}$ indicates that the value from the target level B3LYP/6-311+G(3df,2p)/(75 302) was used

Primary DFT calculation

\begin{tabular}{llllll}
\hline Functional & Basis & Grid & MEAN & MAX & NO \\
\hline SVWN & $2^{\circ}$ & $2^{\circ}$ & 5424 & 22762 & 257 \\
BLYP & $2^{\circ}$ & $2^{\circ}$ & 168 & 589 & 254 \\
EDF1 & $2^{\circ}$ & $2^{\circ}$ & 112 & 804 & 250 \\
$2^{\circ}$ & $6-31 \mathrm{G}$ & $2^{\circ}$ & 402 & 1953 & 257 \\
$2^{\circ}$ & $6-31 \mathrm{G}(d)$ & $2^{\circ}$ & 231 & 913 & 257 \\
$2^{\circ}$ & $6-311 \mathrm{G}(d)$ & $2^{\circ}$ & 78 & 402 & 254 \\
$2^{\circ}$ & $2^{\circ}$ & SG-0 & 2.6 & 22 & 49 \\
BLYP & $2^{\circ}$ & SG-0 & 168 & 589 & 253 \\
$2^{\circ}$ & $6-31 \mathrm{G}(d)$ & SG-0 & 234 & 894 & 257 \\
BLYP & $6-31 \mathrm{G}(d)$ & $2^{\circ}$ & 415 & 1078 & 257 \\
BLYP & $6-31 \mathrm{G}(d)$ & SG-0 & 420 & 1054 & 257 \\
EDF1 & $6-31 \mathrm{G}(d)$ & SG-0 & 130 & 518 & 256
\end{tabular}

\begin{tabular}{|c|c|c|c|c|c|c|c|}
\hline \multicolumn{4}{|c|}{ DFPC (Type A) } & \multicolumn{4}{|c|}{ DFPC (Type B) } \\
\hline MEAN & MAX & WC & $\mathrm{NO}$ & MEAN & MAX & WC & $\mathrm{NO}$ \\
\hline 30 & 73 & $\mathrm{C}_{8} \mathrm{H}_{18}$ & 256 & 2.9 & 21 & $\mathrm{SF}_{6}$ & 63 \\
\hline 5.9 & 15 & $\mathrm{C}_{2} \mathrm{~F}_{6}$ & 178 & 0.4 & 3.0 & CINO & 0 \\
\hline \multirow[t]{4}{*}{4.5} & 12 & Azulene & 137 & 0.6 & 4.4 & $\mathrm{SO}_{2} \mathrm{Cl}_{2}$ & 1 \\
\hline & & & & 26 & 340 & $\mathrm{PCl}_{5}$ & 189 \\
\hline & & & & 1.3 & 21 & $\mathrm{PCl}_{5}$ & 16 \\
\hline & & & & 1.6 & 31 & $\mathrm{PCl}_{5}$ & 23 \\
\hline \multirow[t]{6}{*}{0.005} & 0.05 & $\mathrm{SO}_{2} \mathrm{Cl}_{2}$ & 0 & 0.00006 & 0.0007 & $\mathrm{~B}_{2} \mathrm{H}_{4}$ & 0 \\
\hline & & & & 0.4 & 3.0 & CINO & 0 \\
\hline & & & & 1.3 & 21 & $\mathrm{PCl}_{5}$ & 16 \\
\hline & & & & 1.2 & 12 & $\mathrm{PCl}_{5}$ & 8 \\
\hline & & & & 1.2 & 12 & $\mathrm{PCl}_{5}$ & 8 \\
\hline & & & & 1.3 & 11 & $\mathrm{PCl}_{5}$ & 8 \\
\hline
\end{tabular}


49 outliers, but the dual grid approaches remove all of these and yield maximum errors of $0.05 \mathrm{~kJ} / \mathrm{mol}(\mathrm{DG}(\mathrm{A}))$ or $0.0007 \mathrm{~kJ} / \mathrm{mol}(\mathrm{DG}(\mathrm{B}))$. The benefits of this are clear; the SG-0 grid has around one fifth of the number of points of the "Fine Grid" and this significantly lowers the cost of the selfconsistent primary calculations. It is beyond the scope of this Paper to construct and explore even smaller primary grids for dual grid calculations, but our results indicate that there is ample scope for this.

For the molecules considered, the use of SG-0 as a primary grid has very little impact on the final energies. However, we note that all the molecules in the data set are compact and, for more diffuse systems such as anions and loose complexes, a primary grid with a larger radial extent may be necessary. ${ }^{36}$

\subsection{Double jumping}

Having considered perturbations in each of the functional, basis and grid dimensions separately, we now turn to double jump methods where we consider changes in two dimensions. Based on the results above, we tested three primary levels that are formed from BLYP, 6-31G $(d)$ and SG-0 primary quantities, namely BLYP/6-311+G(3df,2p)/SG-0, B3LYP/6-31G(d)/SG-0 and $\mathrm{BLYP} / 6-31 \mathrm{G}(d) /(75,302)$.

The results for the double jump methods in Table 1 show that the errors for methods that jump in the grid dimension (i.e. DFDG and DBDG) are identical to their non-grid counterparts (DF and DB). This reconfirms that the error introduced by the grid jump is very small. Interestingly, the error for the DFDB method $(1.2 \mathrm{~kJ} / \mathrm{mol})$ is actually smaller than the associated DB method $(1.3 \mathrm{~kJ} / \mathrm{mol})$. This indicates a strong non-additivity of the errors in some of the multi-jump methods. From an accuracy point of view it is clear that the DFDG methods is the best.

\subsection{Triple jumping}

Finally, we consider full DFPC, where we allow changes in all three dimensions. When doing so, it is prudent to ensure that the treatment is balanced and that the DFPC error is not dominated by the error in any single dimension. If it were, further savings could be made by using cheaper primary levels in the other two dimensions. Based on the results above,

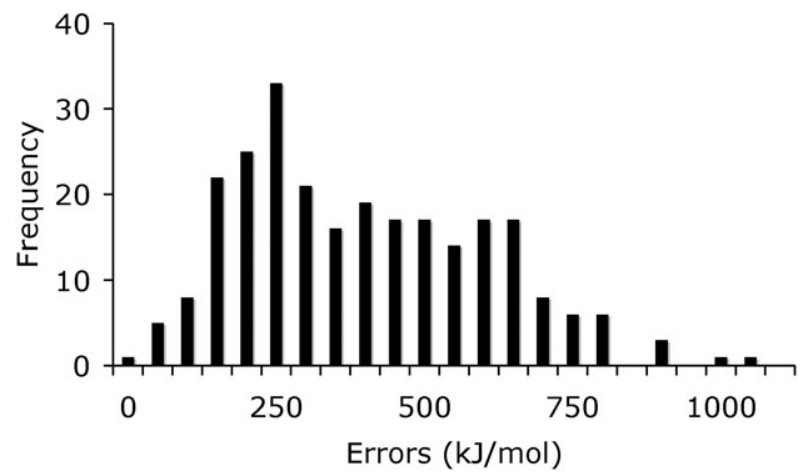

Fig. 2 Errors of primary energies relative to secondary energies for the neutral G3 molecules. The primary and secondary levels were BLYP/ 6-31G(d)/SG-0 and B3LYP/6-311+G(3df,2p)/(75,302), respectively. we tested two primary levels, namely $\operatorname{EDF} 1 / 6-31 \mathrm{G}(d) / \mathrm{SG}-0$ and BLYP/6-31G $(d) / \mathrm{SG}-0$.

The results in the final two rows of Table 1 show that the DFPC errors are very small, with means near $1.3 \mathrm{~kJ} / \mathrm{mol}$ and maxima around $12 \mathrm{~kJ} / \mathrm{mol}$. It is surprising to note that, whereas the EDF1 primary calculations are much more accurate than the BLYP ones, this advantage does not persist in the resulting DFPC energies. This suggests that the EDF1 and BLYP densities are similar.

The error histograms in Fig. 2 and 3 provide further detail on the performance of the DFPC scheme. Fig. 2 shows the errors of the primary $\mathrm{BLYP} / 6-31 \mathrm{G}(d) / \mathrm{SG}-0$ energies, which possess a mode near $250 \mathrm{~kJ} / \mathrm{mol}$ and range up to $1054 \mathrm{~kJ} / \mathrm{mol}$. In contrast, Fig. 3 reveals that the distribution of the DFPC errors is more than two orders of magnitude narrower, and almost entirely concentrated between 0 and $4 \mathrm{~kJ} / \mathrm{mol}$. Most of the eight outliers- $\mathrm{PCl}_{5}, \mathrm{SO}_{2} \mathrm{Cl}_{2}, \mathrm{POCl}_{3}, \mathrm{P}_{4}, \mathrm{~S}_{2} \mathrm{Cl}_{2}, \mathrm{SiCl}_{4}$, $\mathrm{PCl}_{3}$, and $\mathrm{S}_{2} \mathrm{O}$ - are hypervalent and their relatively large DFPC errors arise because the primary basis, 6-31G $(d)$, struggles to describe the important $\mathrm{d}$ orbital participation in these systems.

\subsection{Computational cost}

To assess the cost of DFPC, we compared $\{$ BLYP, B3LYP $\}$ / $\{6-31 \mathrm{G}(d), \quad 6-311+\mathrm{G}(3 d f, 2 p)\} /\{\mathrm{SG}-0, \quad(75,302)\}$ calculation times with those of iterative $\mathrm{B} 3 \mathrm{LYP} / 6-311+\mathrm{G}(3 d f, 2 p) /(75,302)$ calculations for threonine $\left(\mathrm{C}_{4} \mathrm{H}_{9} \mathrm{NO}_{3}\right)$, caffeine $\left(\mathrm{C}_{8} \mathrm{H}_{10} \mathrm{~N}_{4} \mathrm{O}_{2}\right)$, porphine $\left(\mathrm{C}_{20} \mathrm{H}_{14} \mathrm{~N}_{4}\right)$ and the guanine $\cdots$ cytosine $\left(\mathrm{C}_{9} \mathrm{H}_{10} \mathrm{~N}_{8} \mathrm{O}_{2}\right)$ DNA base pair in the Watson-Crick arrangement, abbreviated as GCwc. The structures of threonine, caffeine and porphine were optimized at the $\mathrm{HF} / 6-31 \mathrm{G}(d)$ level and the structure of GCwc was obtained from the literature. ${ }^{37}$ Because DFPC is related to dual basis DFT (DBDFT) techniques, we also present results for DBDFT for comparison. Our secondary calculations used the Q-CHEM default initial guess (superposition of atomic densities), convergence threshold $\left(10^{-5}\right)$ and SCF algorithm (DIIS). A tighter integral threshold of $10^{-10}$ was used to eliminate numerical issues.

Although the full secondary SCF calculations for these systems required only 8-9 cycles, the DFPC calculations nonetheless show substantial speedups over the iterative calculations. Table 2 shows the DFPC calculations take

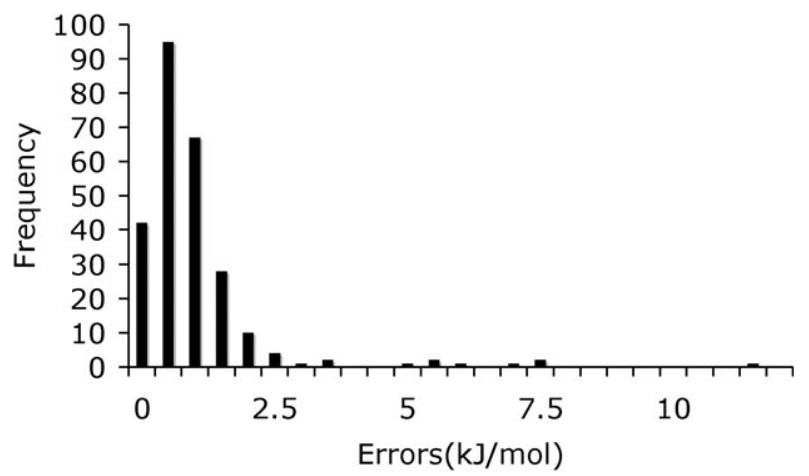

Fig. 3 Errors of DFPC energies relative to secondary energies for the neutral G3 molecules. The primary and secondary levels were BLYP/ 6-31G $(d) / \mathrm{SG}-0$ and $\mathrm{B} 3 \mathrm{LYP} / 6-311+\mathrm{G}(3 d f, 2 p) /(75,302)$, respectively. 
Table 2 Errors $(\mathrm{kJ} / \mathrm{mol})$ and timing ratios for functional $(\mathrm{DF}(\mathrm{B}))$, basis (DB), grid (DG(A)), functional-basis (DFDB), functional-grid (DFDG), and basis-grid (DBDG) jumping, along with DFPC and DBDFT. The primary functional, basis and grid values BLYP, 6-31G $(d)$ and SG-0 were used where appropriate, except for DBDFT where the $6-311 \mathrm{G}(d)$ basis was used. In all cases the target secondary level was B3LYP $/ 6-311+\mathrm{G}(3 d f, 2 p) /(75,302)$

\begin{tabular}{llllll}
\hline & & Threonine & Caffeine & Porphine & GCwc \\
\hline DF(B) & Error & 0.7 & 1.5 & 1.1 & 2.4 \\
& $\mathrm{~T}_{\text {DF }} / \mathrm{T}_{\mathrm{S}}$ & 0.66 & 0.73 & 0.74 & 0.79 \\
DB & Error & 2.0 & 1.6 & 1.9 & 2.5 \\
& $\mathrm{~T}_{\text {DB }} / \mathrm{T}_{\mathrm{S}}$ & 0.34 & 0.30 & 0.36 & 0.31 \\
DG(A) & Error & 0.005 & 0.03 & 0.01 & 0.02 \\
& $\mathrm{~T}_{\mathrm{DG}}(\mathrm{A}) / \mathrm{T}_{\mathrm{S}}$ & 0.69 & 0.83 & 0.81 & 0.83 \\
DFDB & Error & 2.7 & 2.1 & 2.6 & 4.4 \\
& $\mathrm{~T}_{\text {DFDB }} / \mathrm{T}_{\mathrm{S}}$ & 0.33 & 0.29 & 0.37 & 0.31 \\
DFDG & Error & 0.8 & 1.6 & 1.2 & 2.4 \\
& $\mathrm{~T}_{\text {DFDG }} / \mathrm{T}_{\mathrm{S}}$ & 0.52 & 0.47 & 0.60 & 0.53 \\
DBDG & Error & 2.0 & 1.5 & 2.0 & 2.5 \\
& $\mathrm{~T}_{\text {DBDG }} / \mathrm{T}_{\mathrm{S}}$ & 0.25 & 0.25 & 0.40 & 0.29 \\
DFPC & Error & 2.7 & 2.1 & 2.7 & 4.4 \\
& $\mathrm{~T}_{\text {DFPC }} / \mathrm{T}_{\mathrm{S}}$ & 0.24 & 0.24 & 0.31 & 0.27 \\
DBDFT & Error & 4.6 & 5.3 & 6.2 & 7.9 \\
& $\mathrm{~T}_{\text {DBDFT }} / \mathrm{T}_{\mathrm{S}}$ & 0.22 & 0.17 & 0.23 & 0.21 \\
\hline & & & & & \\
\hline
\end{tabular}

between $24-31 \%$ of the secondary time and introduce errors of only $2.1-4.4 \mathrm{~kJ} / \mathrm{mol}$ in the total energies. This compares with the DBDFT calculations which, because they ignore quadratic energy corrections from the improved density matrix, require less CPU time (17-23\%) but incur errors twice as large $4.6-7.9 \mathrm{~kJ} / \mathrm{mol}$.

A full breakdown of where the cost savings arise is complicated by the non-additive nature of the savings, however, some insight can be obtained by considering the single jump methods separately. Table 2 shows that jumping in the basis direction provides the largest savings of between $64-70 \%$. This is due to the fact that the smaller basis affects all parts of the energy calculation and not just the calculation of the exchangecorrelation contribution. The next largest saving is from the type $\mathrm{B}$ functional jump, where the reduction in time is far more modest as both the final Fock matrix formation and energy evaluation are carried out in the large secondary basis. Jumping in the grid direction appears to offer the smallest gains in efficiency, but also introduces the smallest errors. Type A functional jumping was not considered as the errors introduced are much larger, and type $\mathrm{B}$ grid jumping was not considered as the additional accuracy over type $\mathrm{A}$ is unnecessary.

Results for the three double jump methods: functional-basis (DFDB), functional-grid (DFDG) and basis-grid DBDG, are also shown in Table 2. Of these the DFDG method is the most accurate, and offers useful cost savings over the DF single jump method. The DFDB method is cheaper than DFDG and yields near identical energies to DFPC, but at a slightly higher cost and is therefore not recommended. The DBDG method has a particularly attractive cost/performance ratio. It is slightly more expensive than DFPC, but yields errors that are more than $25 \%$ smaller.

The choice of which DFPC method to use will ultimately depend on the desired accuracy. However, two methods appear to stand out from a cost/performance point of view. The triple jumping DFPC method offers the greatest potential for for reducing the cost of the calculation and introduces acceptable errors of around 2-4 kJ/mol. The DBDG method has even smaller errors than DFPC and is only slightly more expensive. However, it is worth pointing out that for large molecules, where the Coulomb contribution can be calculated in a linear time, DFT calculations using pure functionals are significantly faster than those using hybrid functionals. In this regime, jumping from a GGA to a hybrid functional is likely to offer more significant time savings.

\subsection{Default primary level of theory}

DFPC is flexible enough to be able to jump between any two combinations of functional, basis and grid. However, one must specify six quantities to define a DFPC calculation completely. To reduce this complexity, it is desirable to have a default primary level of theory as part of the DFPC prescription. This default must balance the cost savings of cheaper primary levels of theory with the errors that are introduced. To this end, we make the following recommendations:

- If the secondary functional is either a hybrid or metaGGA, then the primary functional should be BLYP, otherwise the primary functional should be the same as the secondary.

- If the secondary basis set is larger than 6-31G $(d)$, then the primary basis set should be 6-31G $(d)$. For smaller secondary basis sets the primary should be the same as the secondary.

- The primary grid should be SG-0.

Thus, the recommended primary level of theory is usually $\mathrm{BLYP} / 6-31 \mathrm{G}(d) / \mathrm{SG}-0$. The cost of a DFPC calculation is dominated by the final energy evaluation using the secondary level of theory and there is little potential for computational savings using a cheaper primary level of theory.

\subsection{Geometry optimization and frequency}

Because the DFPC energy is not variationally optimized at the secondary level with respect to the molecular orbital coefficients, the derivatives with respect to these parameters are not zero. Consequently, calculating the exact analytic first derivatives requires solving a single set of coupled-perturbed SCF (CP-SCF) equations. ${ }^{38}$ This incurs a significant additional cost, which defeats the savings obtained in the underlying DFPC single-point calculation. We are pursing an alternative derivative theory which avoids the CP-SCF equations and which is consistent with the underlying DFPC perturbation philosophy. Nonetheless, to demonstrate the potential accuracy that can be obtained for molecular geometries and vibrational frequencies, we have optimized the structure of $\mathrm{H}_{2} \mathrm{O}$ and calculated the vibrational frequencies using finite-difference schemes with a step size of $0.001 \AA$ for the derivatives. Deviations from the secondary level of theory for geometric parameters and frequencies are listed in Table 3. The errors introduced by the use of numerical derivatives are smaller than the resolution of the results in the table.

The DFPC optimized geometric parameters are almost indistinguishable from the benchmark values with differences in the $\mathrm{O}-\mathrm{H}$ bond length and $\mathrm{H}-\mathrm{O}-\mathrm{H}$ bond angle of only a few parts in 10000 . These errors are two orders of magnitude smaller than those in the primary calculation. The vibrational frequencies are more sensitive to the level of theory with errors in the primary calculation being between $4-7 \%$. 
Table 3 Absolute errors in geometry and harmonic vibrational frequencies $\left(\mathrm{cm}^{-1}\right)$ for DFT and DFPC calculations on $\mathrm{H}_{2} \mathrm{O}$. The primary and secondary levels were BLYP/6-31G(d)/SG-0 and B3LYP/6-311+ G(3df,2p)/(75,302), respectively

\begin{tabular}{llll}
\hline & Secondary Benchmark & Primary Error & DFPC Error \\
\hline $\mathrm{O}-\mathrm{H}(\AA)$ & 0.96108 & 0.02 & 0.00008 \\
$\mathrm{H}-\mathrm{O}-\mathrm{H}\left({ }^{\circ}\right)$ & 105.21 & 2.4 & 0.05 \\
$\nu_{1}$ str., $\left(\mathrm{cm}^{-1}\right)$ & 3821 & 258 & 4 \\
$\nu_{2}$ bend, $\left(\mathrm{cm}^{-1}\right)$ & 1628 & 58 & 6 \\
$\nu_{3}$ a. str., $\left(\mathrm{cm}^{-1}\right)$ & 3923 & 237 & 3 \\
\hline
\end{tabular}

The corresponding errors for the DFPC calculation are only a few parts in 1000 and also span a smaller range, indicating a more reliable level of theory. It is clear from these results that, as long as they can be computed efficiently, DFPC derivatives are very accurate.

\section{Conclusions}

We have examined several perturbative corrections that use the results of a low-cost primary SCF calculation to estimate the energy of a more expensive secondary calculation. Our methods, which can be viewed as vertices on a cube whose dimensions represent the functional, basis and grid, all offer time savings compared to the full iterative secondary calculation.

We have tested the accuracy of our DFPC approaches by computing total energies of the 257 neutral molecules in the G3 set. The $\{$ BLYP, B3LYP $\} /\{6-31 \mathrm{G}(d), 6-311+\mathrm{G}(3 d f, 2 p)\} /$ $\{\mathrm{SG}-0,(75,302)\}$ level of DFPC yields energies with a mean absolute error of only $1.2 \mathrm{~kJ} / \mathrm{mol}$, relative to full iterative B3LYP/6-31 1+G(3df,2p)/(75,302) calculations. Moreover, such calculations on threonine, caffeine, porphine and GCwc take around a quarter of the time of the secondary calculations.

Although cheaper primary levels of theory are possible, our results indicate that local density functionals such as SVWN are not accurate enough and unpolarized primary basis sets also introduce unacceptable errors. The choice of the primary grid is much less important and, although we have used SG-0 here, it should be possible to use even smaller grids without significantly affecting the DFPC energies. For these reasons we recommend the use of $\mathrm{BLYP} / 6-31 \mathrm{G}(d) / \mathrm{SG}-0$ for the primary level of theory in most cases.

Reduced variants of DFPC, where jumps are made in only one or two of the dimensions, were also investigated. These methods offer a variety of cost/performance points and the DBDG method is particularly attractive as it yields much more accurate energies than DFPC at only a slightly increased cost. Performance comparisons with related DBDFT scheme show that, using the recommended primary levels of theory, DFPC is slightly more expensive than DBDFT but introduces errors that are only half large.

Using numerical derivatives, we have considered the accuracy of DFPC for geometry optimizations and frequency calculations. The preliminary results are in very good agreement with the benchmark values and provides motivation for developing an analytic gradient theory that avoids solving the expensive CP-SCF equations.

We have focused on hybrid density functionals for our secondary calculation, but our approach can also be applied to the more recent meta-generalized gradient approximation
(meta-GGA) functionals, which explicitly depend on the kinetic energy density. Although more accurate, these functionals are even more expensive than hybrids and show an increased sensitivity to the choice of integration grid. ${ }^{39}$ Our DFPC scheme has the potential to alleviate these problems.

\section{Acknowledgements}

P.M.W.G. thanks the Australian Research Council for funding (Grants No. DP0771978 and DP0984806) and the NCI National Facility for a generous allocation of supercomputer resources. J.D. thanks the ANU/RSC for a PhD scholarship.

\section{References}

1 R. G. Parr and W. Yang, Density Functional Theory of Atoms and Molecules, Oxford University Press, Oxford, 1989.

2 W. Koch and M. C. Holthausen, A Chemist's Guide to Density Functional Theory, Wiley-VCH, Oxford, 2001.

3 B. G. Johnson, P. M. W. Gill, J. A. Pople and D. J. Fox, Chem. Phys. Lett., 1993, 206, 239.

4 P. M. W. Gill, Adv. Quantum Chem., 1994, 25, 141.

5 C. A. White, B. G. Johnson, P. M. W. Gill and M. Head-Gordon, Chem. Phys. Lett., 1994, 230, 8.

6 J. P. Dombroski, S. W. Taylor and P. M. W. Gill, J. Phys. Chem., 1996, 100, 6272.

7 L. Fusti-Molnar and P. Pulay, J. Chem. Phys., 2002, 117, 7827.

8 L. Fusti-Molnar and J. Kong, J. Chem. Phys., 2005, 122, 074108.

9 T. Nakajima and K. Hirao, J. Chem. Phys., 2006, 124, 184108.

10 D. J. Tozer, M. E. Mura, R. D. Amos and N. C. Handy, AIP Conf. Proc., 1995, 330, 3.

11 S. Huzinaga and K. Hirao, J. Chem. Phys., 1977, 66, 2157.

12 K. Hirao and S. Huzinaga, Chem. Phys. Lett., 1977, 45, 55.

13 S. Havriliak and H. F. King, J. Am. Chem. Soc., 1983, 105, 4.

14 S. Havriliak, T. R. Furlani and H. F. King, Can. J. Phys., 1984, 62, 1336.

15 W. Liang and M. Head-Gordon, J. Phys. Chem. A, 2004, 108, 3206.

16 R. P. Steele, R. A. DiStasio, Jr, Y. Shao, J. Kong and M. Head-Gordon, J. Chem. Phys., 2006, 125, 074108.

17 R. P. Steele, Y. Shao, R. A. DiStasio, Jr and M. Head-Gordon, J. Phys. Chem. A, 2006, 110, 13915.

18 J. Deng, A. T. B. Gilbert and P. M. W. Gill, J. Chem. Phys., 2009, 130, 231101.

19 J. Deng, A. T. B. Gilbert and P. M. W. Gill, J. Chem. Phys., in press.

20 J. A. Pople, P. M. W. Gill and B. G. Johnson, Chem. Phys. Lett., 1992, 199, 557.

21 P. M. W. Gill, B. G. Johnson and J. A. Pople, Chem. Phys. Lett., 1993, 209, 506.

22 C. W. Murray, N. C. Handy and G. J. Laming, Mol. Phys., 1993, 78, 997-1014.

23 V. I. Lebedev, Sibirsk. Mat. Zh., 1977, 18, 132.

24 M. J. Frisch, G. W. Trucks, H. B. Schlegel, G. E. Scuseria, M. A. Robb, J. R. Cheeseman, G. Scalmani, V. Barone, B. Mennucci, G. A. Petersson, H. Nakatsuji, M. Caricato, X. Li, H. P. Hratchian, A. F. Izmaylov, J. Bloino, G. Zheng, J. L. Sonnenberg, M. Hada, M. Ehara, K. Toyota, R. Fukuda, J. Hasegawa, M. Ishida, T. Nakajima, Y. Honda, O. Kitao, 
H. Nakai, T. Vreven, J. A. Montgomery Jr, J. E. Peralta, F. Ogliaro, M. Bearpark, J. J. Heyd, E. Brothers, K. N. Kudin, V. N. Staroverov, R. Kobayashi, J. Normand, K. Raghavachari, A. Rendell, J. C. Burant, S. S. Iyengar, J. Tomasi, M. Cossi, N. Rega, J. M. Millam, M. Klene, J. E. Knox, J. B. Cross, V. Bakken, C. Adamo, J. Jaramillo, R. Gomperts, R. E. Stratmann, O. Yazyev, A. J. Austin, R. Cammi, C. Pomelli, J. W. Ochterski, R. L. Martin, K. Morokuma, V. G. Zakrzewski, G. A. Voth, P. Salvador, J. J. Dannenberg, S. Dapprich, A. D. Daniels, O. Farkas, J. B. Foresman, J. V. Ortiz, J. Cioslowski and D. J. Fox, GAUSSIAN 09, Gaussian, Inc., Wallingford, CT, 2009.

25 S. T. Brown and J. Kong, Chem. Phys. Lett., 2005, 408, 395.

26 Y. Shao, L. Fusti-Molnar, Y. Jung, J. Kussmann, C. Ochsenfeld, S. T. Brown, A. T. B. Gilbert, L. V. Slipchenko, S. V. Levchenko, D. P. O'Neill, R. A. DiStasio, Jr, R. C. Lochan, T. Wang, G. J. O. Beran, N. A. Besley, J. M. Herbert, C. Y. Lin, T. V. Voorhis, S. H. Chien, A. Sodt, R. P. Steele, V. A. Rassolov, P. E. Maslen, P. P. Korambath, R. D. Adamson, B. Austin, J. Baker, E. F. C. Byrd, H. Dachsel, R. J. Doerksen, A. Dreuw, B. D. Dunietz, A. D. Dutoi, T. R. Furlani, S. R. Gwaltney, A. Heyden, S. Hirata, C. P. Hsu, G. Kedziora, R. Z. Khalliulin, P. Klunzinger, A. M. Lee, M. S. Lee, W. Z. Liang, I. Lotan, N. Nair, B. Peters, E. I. Proynov, P. A. Pieniazek, Y. M. Rhee, J. Ritchie, E. Rosta,
C. D. Sherrill, A. C. Simmonett, J. E. Subotnik, H. L. Woodcock III, W. Zhang, A. T. Bell, A. K. Chakraborty, D. M. Chipman, F. J. Keil, A. Warshel, W. J. Hehre, H. F. Schaefer III, J. Kong, A. I. Krylov, P. M. W. Gill and M. Head-Gordon, Phys. Chem. Chem. Phys., 2006, 8, 3172.

27 L. A. Curtiss, K. Raghavachari, P. C. Redfern and J. A. Pople, J. Chem. Phys., 2000, 112, 7374.

28 J. C. Slater, Phys. Rev., 1951, 81, 385.

29 S. H. Vosko, L. Wilk and M. Nusair, Can. J. Phys., 1980, 58, 1200.

30 A. D. Becke, Phys. Rev. A, 1988, 38, 3098.

31 C. Lee, W. Yang and R. G. Parr, Phys. Rev. B, 1988, 37, 785.

32 R. D. Adamson, P. M. W. Gill and J. A. Pople, Chem. Phys. Lett., 1998, 284, 6.

33 R. S. Grev and H. F. Schaefer III, J. Chem. Phys., 1989, 91, 7305.

34 P. M. W. Gill and S. H. Chien, J. Comput. Chem., 2003, 24, 732.

35 S. H. Chien and P. M. W. Gill, J. Comput. Chem., 2006, 27, 730.

36 J. Gräfenstein and D. Cremer, J. Chem. Phys., 2007, 127, 164113.

37 P. Jurecka, J. Sponer, J. Cerny and P. Hobza, Phys. Chem. Chem. Phys., 2006, 8, 1985-1993.

38 Y. Yamaguchi, O. Osamura, J. D. Goddard and H. F. Schaefer, $A$ New Dimension to Quantum Mechanics. Analytical Derivative Methods in Ab Initio Molecular Electronic Structure Theory, Oxford University Press, Oxford, 1994.

39 S. E. Wheeler and K. N. Houk, J. Chem. Theory Comput., 2010, 6, 395. 